\title{
Cerebral Malaria as a Complication of Plasmodium Vivax
}

\author{
Dr. Shashank Shekhar ${ }^{1}$, Dr. Dipti Neha ${ }^{2}$, Dr.Amar Verma ${ }^{3}$ \\ ${ }^{1}$ Junior Resident, ${ }^{2}$ Senior Resident, ${ }^{3}$ Associate Professor \\ Department of Pediatrics and Neonatology, Rajendra Institute of Medical Sciences (RIMS), Ranchi, Jharkhand
}

\begin{abstract}
Background: Cerebral malaria is a diffuse encephalopathy characterised by unarousable coma of more than 30 minutes duration associated with or without seizures. Usually cerebral malaria is caused by P. falciparum, but rarely it could be the presenting complication or occurring during the course of $P$. vivax. P.vivax is responsible for benign, uncomplicated disease. Newer reports are now suggestive of severe manifestations of P.vivax malaria.

Objective: To assess the natural history, associated complications, response to treatment and outcome of cerebral malaria due to $P$. vivax.

Methodology: This study is a hospital based prospective study conducted from September 2015 to August 2016 in Department of Paediatrics and Neonatology, Rajendra Institute of Medical Sciences, Ranchi. In this study, 110 confirmed cases of severe complicated malaria diagnosed on the basis of blood smear examination were included.

Results: Out of 110 cases of severe complicated malaria admitted during this period, 35 patients (31.8\%) presented with cerebral malaria of which 23 were due to $P$. falciparum, 8 due to $P$. vivax and rest 8 were due to mixed infections. The predominant clinical manifestations were fever, seizures and altered sensorium. Main associated complications were severe anaemia (62\%) and thrombocytopenia (50\%). All the patients responded to IV artesunate. Follow-up evaluation after one month showed no residual neurological deficit.

Conclusion: P. vivax infection is usually presumed to cause only uncomplicated malaria but in the last few years, there have been many reports regarding cerebral vivax malaria, the exact pathological mechanism is still elusive. Patients of vivax malaria should be monitored for occurrence of different complications as their early detection and treatment can be life saving.
\end{abstract}

Keywords: $P$. vivax, cerebral malaria

\section{Introduction}

Malaria is one of the most important parasitic diseases of humans. Approximately $40 \%$ of the world's population lives in regions where malaria transmission is endemic. The World Health Organization (WHO) estimated that 214 million cases of malaria occurred globally and approximately 438,000 deaths were recorded in 2015. ${ }^{1}$ Of the various Plasmodium species affecting humans, Plasmodium vivax malaria is long considered to have a benign course. It is known for multiple relapses; but the typical complications seen with falciparum malaria are not found with vivax monoinfection. ${ }^{2}$ However in the past few years there is a changing trend in the clinical manifestations of vivax malaria namely severe complicated disease. Cerebral malaria is a diffuse encephalopathy characterised by unarousable coma of more than 30 minutes duration associated with or without seizures. Usually cerebral malaria is caused by P. falciparum, but rarely it could be the presenting complication or occurring during the course of P. vivax. Only 45 cases of cerebral malaria due to $P$. vivax are reported in the scientific literature since $1920 .^{3}$

\section{Methodology}

This study is a hospital based prospective study conducted from September 2015 to August 2016 in the Department of Paediatrics and Neonatology, Rajendra Institute of Medical Sciences, Ranchi. In this study, 110 confirmed cases of severe complicated malaria diagnosed on the basis of blood smear examination were included. Investigations included complete blood count with peripheral blood smear, random blood sugar, serum electrolytes, renal function test and liver function test.

\section{Results}

Out of 110 cases of severe complicated malaria admitted during this period, 35 patients presented with cerebral malaria of which $23(54 \%)$ were due to P. falciparum, $8(23 \%)$ due to P. vivax and rest $8(23 \%)$ were due to mixed infections. (Fig 1) 


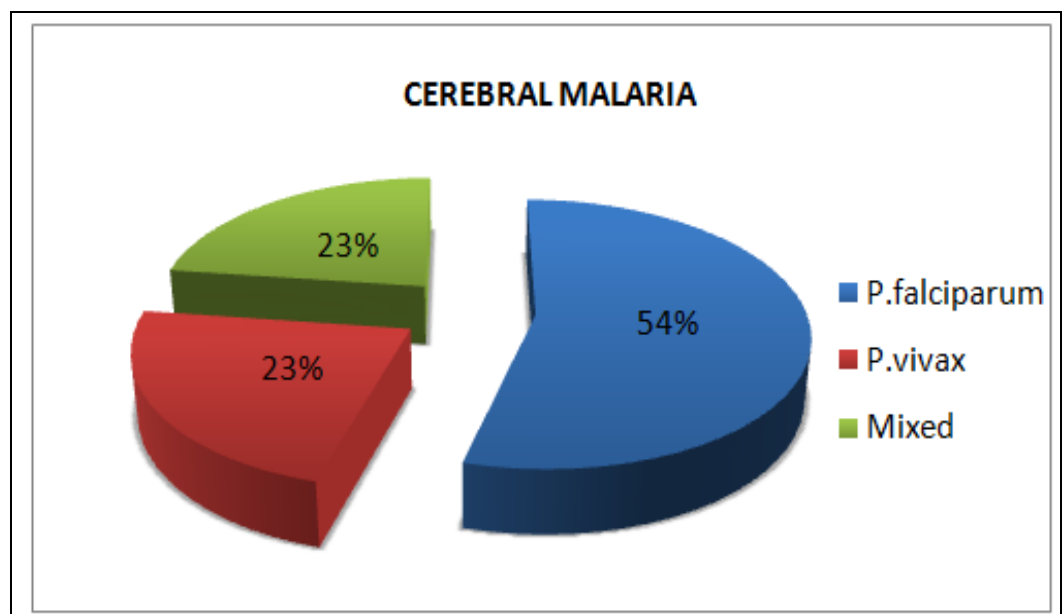

Fig 1: Case Distribution according to species

Out of 8 cases of cerebral malaria due to P.vivax, majority of the cases i.e. 4 cases (50\%) presented on day 5, 2 cases $(25 \%)$ on day 3,1 case (12.5\%) each on day 4 and day 7 . (Fig 2 )

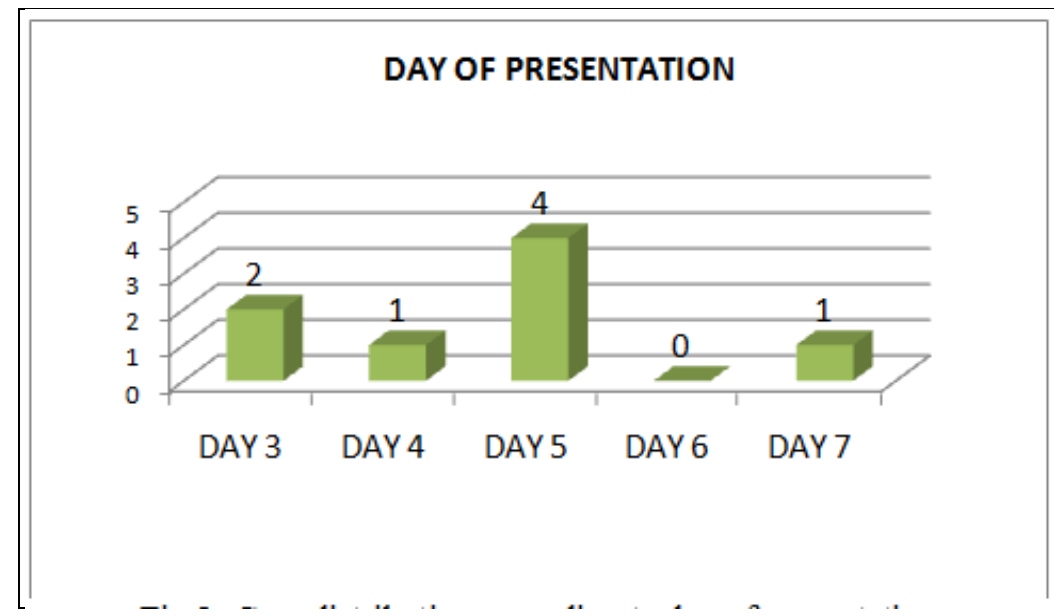

Fig 2: Case distribution according to day of presentation

The predominant clinical manifestations were fever, seizures and altered sensorium which were present in all the 8 cases (100\%). Other clinical manifestations were headache which was present in 6 cases $(75 \%)$, vomiting in 3 cases $(37.5 \%)$. (Fig 3)

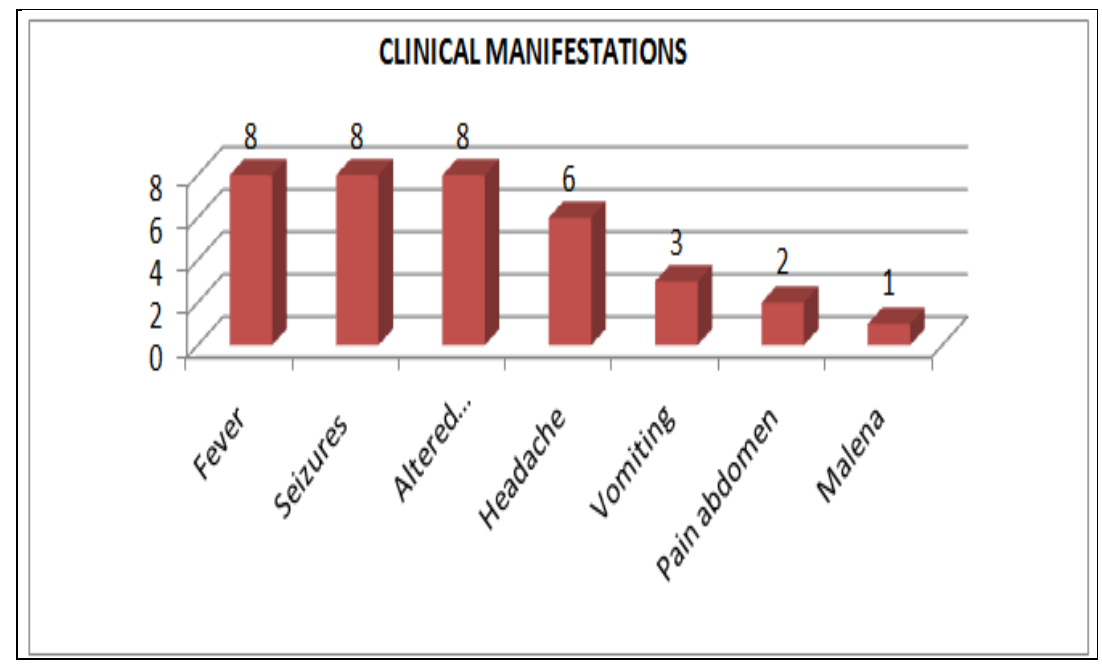

Fig 3: Case distribution according to clinical manifestations 
Complications seen in these patients were: $5(62.5 \%)$ had severe anaemia, $4(50 \%)$ had thrombocytopenia, 2 $(25 \%)$ had jaundice, $2(25 \%)$ patients had hypernatremia and 1(12.5\%) presented with DIC. (Fig 4)

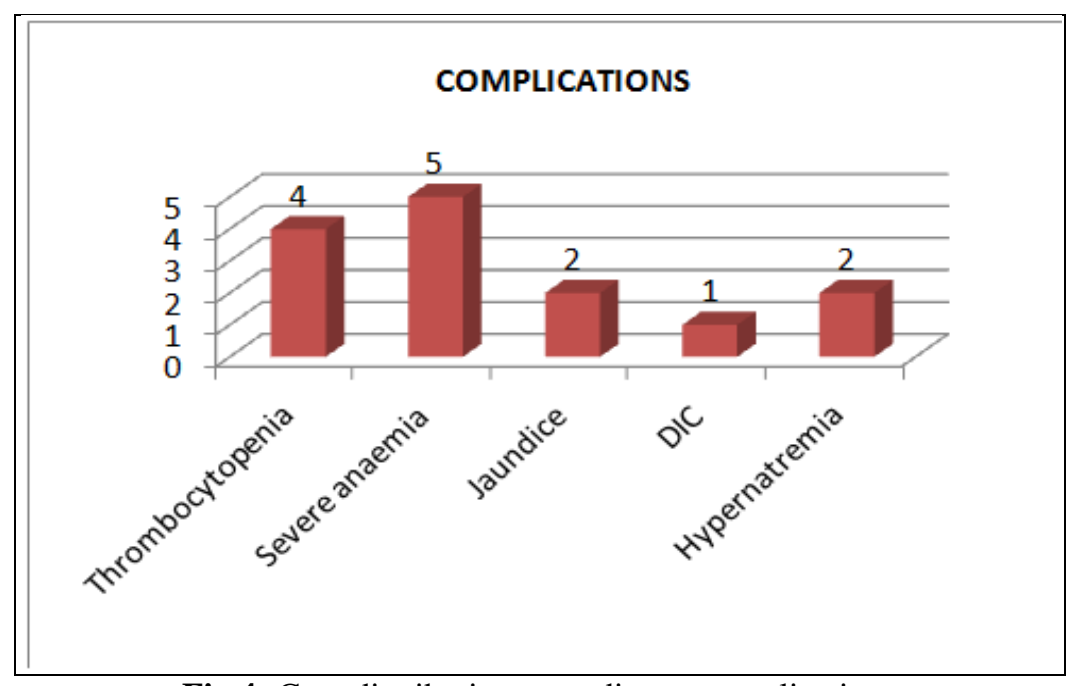

Fig 4: Case distribution according to complications

All the patients were treated with intravenous artesunate and supportive therapy as required. Repeat blood smears were done for parasitic clearance. Out of 8 cases, $4(50 \%)$ cases attained parasitic clearance on Day 3, 2 (25\%) on Day 5 and 1 (12.5\%) each on Day 4 and Day 7. (Fig 5)

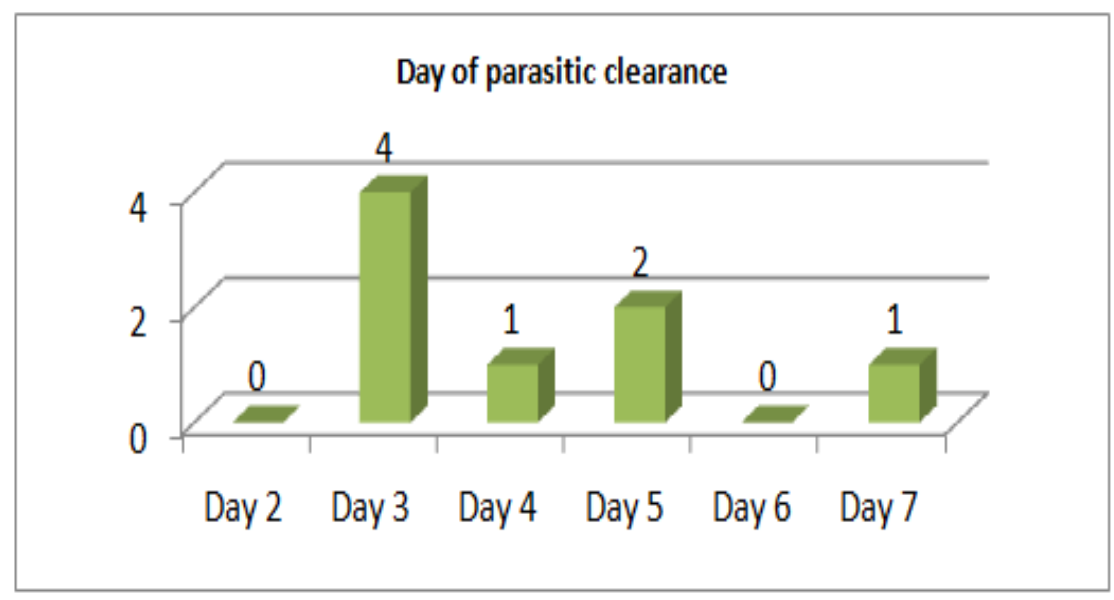

Fig 5: Case distribution according to day of parasitic clearance

All the eight patients were discharged from the hospital in a clinically stable condition and were advised to take primaquine for 14 days. Two of the eight patients had neurological deficits in form of hemiparesis and one of them had aphasia at the time of discharge. Follow-up evaluation after one month showed no residual neurological deficit.

\section{Discussion}

Plasmodium falciparum is considered to be the main cause of severe complicated malaria but Plasmodium vivax is being increasingly recognised as one of the etiological factors for severe complicated malaria. Out of 35 cases of cerebral malaria, 8 cases $(23 \%)$ were due to P. vivax monoinfection. The predominant clinical manifestations were fever, seizures and altered sensorium with mean day of presentation being $4.62 \pm 1.30$ days. The most common complications seen were severe anaemia $(62 \%)$ and thrombocytopenia (50\%). All the patients responded to IV artesunate with mean day of parasitic clearance being around $4.12 \pm 1.45$ days. There was no residual neurological deficit at 1 month of follow up. Organ dysfunction characteristic of $\mathrm{P}$. falciparum infection is unusual in P. vivax illness. P. vivax malaria earlier thought to be benign isn't quite benign any more. 
Cerebral Malaria As A Complication Of Plasmodium Vivax

\begin{tabular}{|l|l|l|l|}
\hline Studies & Age Group & $\begin{array}{l}\text { No. of } \\
\text { Complicated Malaria } \\
\text { Cases }\end{array}$ & $\begin{array}{l}\text { No. Of Cerebral Malaria } \\
\text { Cases Due To P.Vivax }\end{array}$ \\
\hline Charulata S Limaye Et Al, 2012 & Adults & 162 & $12(7.4 \%)$ \\
\hline Rajesh Kumar Et Al, 20145 & $0-15$ Years & 81 & $5(6.1 \%)$ \\
\hline Suman Sarkar Et Al, 2008 & Adults & - & 3 \\
\hline Shoba Philip Et Al, 2015 & Adults & - & 1 \\
\hline Ravi Bhatia Et Al, $2014^{8}$ & 9 Years & - & 1 \\
\hline Singh Et Al, 2014 & Adults & 110 & $19(17.2 \%)$ \\
\hline Present Study & $1-15$ Years & 110 & $8(7.3 \%)$ \\
\hline
\end{tabular}

Fig 6: Cerebral Malaria cases due to P. vivax in different studies

Any patient infected with $\mathrm{P}$. vivax who exhibits severe malaria is presumed to be suffering from mixed infection. However, that may not be always true. As evident from the present study, P. vivax infection can also present as cerebral malaria. Clinical data provided by Kochar et $\mathrm{al}^{10}$, indicates that P. vivax can cause both sequestration related and non sequestration related complications of severe malaria, all of which are commonly associated with $\mathrm{P}$. falciparum infections. The exact pathogenesis however remains elusive.

\section{Conclusion}

P. vivax infection is responsible for $60-70 \%$ of malaria cases in India and it is the most important cause of morbidity. It is usually presumed to cause only uncomplicated malaria but in the last few years, there have been many reports regarding cerebral vivax malaria, the exact pathological mechanism is still elusive. Patients of vivax malaria should be monitored for occurrence of different complications as their early detection and treatment can be life saving.

\section{References}

[1]. World Health Organization. World malaria report 2015. World Health Organization, Geneva, 2015; http://apps.who.int/iris/bitstream/10665/200018/1/9789241565158_eng.pdf.

[2]. World Health Organisation. Severe falciparum malaria. Transactions of the Royal Society of Tropical Medicine and Hygiene 2000:94(Suppl.1):1-90.

[3]. Ozen M, Gungor S, Atambay M, Daldal N. Cerebral malaria owing to Plasmodium vivax: Case report. Ann J Pediatr 2006;26:1414.

[4]. Charulata S Limaye, Vikram A Londhe, ST Nabar. The Study of Complications of Vivax Malaria in Comparison with Falciparum Malaria in Mumbai: JAPI • October 2012 - VOL. 60: 15-18.

[5]. Kumar R, Agarwal D, Kumar P. Severe Plasmodium vivax Malaria in Children: An emerging threat. Journal of Pediatric Sciences. 2014;6:e210

[6]. Suman Sarkar, Prithwis Bhattacharya. Cerebral malaria caused by Plasmodium vivax in adult subjects: Indian J Crit Care Med October-December 2008 Vol 12 Issue 4, 204-5.

[7]. Thomas R, Alexander A, Paul A, Philip S, Rajeev I (2015) Plasmodium Vivax Cerebral Malaria - A Rare Cause of Multi Organ Dysfunction. J Anesth Crit Care Open Access 3(3): 00097. DOI: 10.15406/jaccoa.2015.03.00097

[8]. Ravi Bhatia , Gunjan Bhatia. Cerebral malaria following P. Vivax infection- A case report: Indian Journal of Basic and Applied Medical Research; September 2014: Vol.-3, Issue- 4, P. 356-358

[9]. Singh J, Purohit B, Desai A, Savardekar L, Shanbag P, Kshirsagar N et.al Clinical manifestations ,

[10]. treatment and outcome of hospitalized patients with Plasmodium Vivax malaria in two Indian states : a retrospective study. Malaria Research and treatment, Volume 2013, Article ID 341862.

[11]. Kochar DK, Das A, Kochar SK et.al Severe Plasmodium Vivax Malaria: a report on serial cases from Bikaner in north western India. Am J Trop Med Hyg 2009; 80: 194-8. 